\title{
A Golden Opportunity and Call for Action for Whole of Family Lifestyle Change to Significantly Reduce the Risk of Development of Severe COVID-19
}

\author{
Gary M Leong* \\ Department of Paediatrics, Clinical Lead Paediatric Diabetes and Family Metabolic Health Services, University of Sydney, Nepean Hospital, Australia \\ *Corresponding author: Gary M Leong, The Children's Clinic; the Charles Perkins Centre Nepean Research Hub, The University of \\ Sydney and the Department of Paediatrics, Clinical Lead Paediatric Diabetes and Family Metabolic Health Services, Nepean Hospital, \\ Kingswood, New South Wales, Australia.
}

To Cite This Article: Gary M Leong. A Golden Opportunity and Call for Action for Whole of Family Lifestyle Change to Significantly Reduce the Risk of Development of Severe COVID-19. 2020 - 9(5). AJBSR.MS.ID.001428. DOI: 10.34297/AJBSR.2020.09.001428.

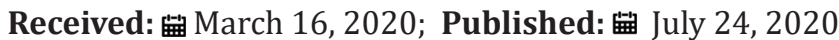

\section{Opinion}

To say COVID-19 has turned every country on its head, causing unprecedented public health, economic and societal pressure, is a colossal understatement. With no known therapies or a preventative vaccine, the novel Severe Acute Respiratory Syndrome Corona Virus 2 (SARS-CoV-2) strain is responsible globally to date for over three hundred thousand deaths and $>4$ million infected individuals and a global economic and social shutdown [1]. The lack of an effective preventative vaccine highlights the importance in understanding what modifiable lifestyle factor(s) that predispose any one individual to the development of severe COVID-19 disease, including the risk of mechanical ventilation and death.

Recent analyses of adults $<60$ years of age hospitalized with COVID-19 in the USA [2] and in France [3], and many other countries, clearly indicate individuals with severe obesity with a BMI $>35 \mathrm{~kg} /$ $\mathrm{m} 2$ have a significantly greater risk (over $7 \mathrm{x}$ risk compared to individuals with a BMI $<25 \mathrm{~kg} / \mathrm{m} 2$ ) of mechanical ventilation, with almost $50 \%$ of hospitalized patients with COVID-19 being obese $[2,3]$. This increased risk of obesity to the development severe COVID-19 may be due to many underlying pathological effects of obesity. These include effects on the cardiorespiratory and immune systems and appear independent of the adverse effects of diabetes. Of relevance to COVID-19 are the effects of obesity on a) proinflammation; b) reduced respiratory reserve, including central and peripheral obstructive sleep apnoea and obesity-relatedhypoventilation syndrome; c) impairment of immune function secondary to nutritional micronutrient deficiencies, and d) lack of adequate physical activity and sleep [4,5].
In keeping with these nutritional deficits in individuals with obesity, a recent intriguing report suggested that vitamin D deficiency may be a possible modifiable risk factor and that vitamin D supplementation may prevent or ameliorate severe COVID-19 [6]. This report is consistent with the known role of vitamin D as an anti-inflammatory agent and highlights a potential important role for this hormone on positively influencing COVID-19 outcomes. Moreover, it highlights the importance of connection of families to more outdoor activities together in natural settings, as clearly adequate and safe sunshine exposure through UVB-light exposure on Vitamin D pre-hormones in the skin is the easiest and most reliable source of vitamin D for children and adults alike [7]. Recent alarming reports have appeared of younger individuals $<50$ years of age being severely affected by COVID-19-induced thrombotic strokes [8] and COVID-19 affecting young children with a Kawasaki-like pro-inflammatory illness called pediatric multi system inflammatory syndrome (PMIS) [9].

The influences of modifiable lifestyle factors in these rare non-respiratory manifestations of severe COVID-19 remain to be determined. In the Unites States and Australia alike, the obesity COVID-19 link is troubling given that up to two-thirds of the adult population and a quarter to a third of children and adolescents fall into the overweight or obese range. Obesity also disproportionately affects many families from more disadvantaged ethnic minorities, who are generally ill-equipped to deal with the health and economic challenges of obesity, yet alone that of a COVID-19 infection [10]. The development of a safe and effective vaccine against SARS-CoV-2, 
though being undertaken at unprecedented breakneck speed, remains elusive. Thus in my opinion, as a practicing pediatrician and endocrinologist dealing on a daily basis with children and their families with severe obesity, it seems apparent and urgent to me, that the next $12-24$ months represent a golden opportunity for positive family health change and development of a communitywide public health campaign. This truly golden opportunity to motivate families to make whole of family lifestyle changes aimed at moving children, parents, and grandparents alike to a healthier weight and life should not be lost. I call upon all individuals and especially parents, and my health professional colleagues, to take on this 2020 family health challenge and seek the help of a supportive relative, friend and/or health professional to help you and your family start this positive life journey for a healthier weight and better mental and physical well-being and connection to your community.

Dr Gary Leong, MBBS, FRACP, PhD Pediatric endocrinologist and author of the book: "Ride to Life: a no-nonsense program for breaking your family's cycle of obesity, and connecting to a healthier, happier life." and founder of the "2020 Family Health Challenge". Join the challenge at

\section{https://2020familyhealthchallenge.com}

\section{Reference}

1. https://www.worldometers.info/coronavirus
2. Lighter J, Phillips M, Stephanie Sterling, Diane Johnson, Anna Stachel, et al. Obesity in patients younger than 60 years is a risk factor for Covid-19 hospital admission. Clinical Infectious Diseases, ciaa415.

3. Simonnet A, Chetboun M, Julien Poissy, Violeta Raverdy, Jerome Noulette, et al. High prevalence of obesity in severe acute respiratory syndrome coronavirus-2 (SARS-CoV-2) requiring invasive mechanical ventilation. Obesity.

4. Heymsfield SB, Wadden TA (2017) Mechanisms, Pathophysiology, and Management of Obesity N Engl J Med 376(3): 254-266.

5. Chandra RK (1997) Nutrition and the immune system: an introduction. Am J Clin Nutr 66(2): 460S-463S.

6. Grant WB, Henry Lahore, Sharon L McDonnell, Carole A Baggerly, et al. (2020) Evidence that vitamin D supplementation could reduce risk of influenza and COVID-19 infections and deaths. Nutrients 12(4): 988.

7. Louv R. The Children and Nature Network https://www. childrenandnature.org.

8. Oxley TJ, J Mocco, Shahram Majidi, Christopher P Kellner, Hazem Shoirah, et al. (2020) Large-vessel stroke as a presenting feature of Covid-19 in the young. N Eng J Med 14: 382(20): e60.

9. Chao JY, Jacqueline Weingarten, Henry M Ushay, Michael D Cabana, Shivanand S Medar, et al. (2020) Clinical Characteristics and Outcomes of Hospitalized and Critically Ill Children and Adolescents with Coronavirus Disease 2019 (COVID-19) at a Tertiary Care Medical Center in New York City. J Pediatr 223: 14-19.

10. (2017) Global Burden of Disease (GBD) 2015 Obesity Collaborators. Health effects of overweight and obesity in 195 countries over 25 years. The N Engl J Med 377: 13-27. 